\title{
¿CUÁNTAS ESPECIES DE CABALLO HUBO EN UNA SOLA LOCALIDAD DEL PLEISTOCENO MEXICANO?
}

\author{
María Teresa ALBERDI ${ }^{1}$, Joaquín ARROYO- \\ CABRALES ${ }^{2}$ y Oscar J. POLACO ${ }^{2}$ \\ ${ }^{1}$ Museo Nacional de Ciencias Naturales, CSIC, José Gutiérrez Abascal 2, \\ 28006-Madrid (España). E-mail: malberdi@mncn.csic.es \\ ${ }^{2}$ Instituto Nacional de Antropología e Historia, Moneda 16 (Col. Centro). \\ 06060-México DF. E-mail: arromatu@hotmail.com
}

Alberdi, M. T., Arroyo-Cabrales, J. y Polaco, O. J. 2003. ¿Cuántas especies de caballo hubo en una sola localidad del Pleistoceno Mexicano? [How many species of horse are there in a single Local Fauna of Mexican Pleistocene?] Revista Española de Paleontología, 18 (2), 205-212. ISSN 0213-6937.

\begin{abstract}
In the past, there has been much discussion about the number of species of horse, Equus (Perissodactyla, Equidae) in the Pleistocene deposits of México. From one up to 10 species have been allocated to a single locality at a time. Here we report on the species occurring in the late Pleistocene archaeological site of El Cedral, San Luis Potosí. The site is located in the scrubland of the Mexican Plateau, and the deposit formed inside an old spring. It was dug in the 1970s and 1980s, resulting in the presence of evidence of human activity at around 30,000 years BP, and with a rich mammal fauna that included more than 5,000 bones, including species like vole (temperate forests inhabitant) and tapir (tropical forest) that do not occur any close of the locality at present. A large sample of cranial and postcranial bones was available for study, including teeth and metapodials, allowing to describe the most important characteristic for the horses groups from the locality. Uni- and multivariate statistical analyses were applied to the data, and results are discussed in regard to taxonomical status of the group. The presence of a large and a small sized species was recorded, with some outliers in between those two sizes. For the large horse the name Equus mexicanus is assigned, while Equus conversidens seems to be the correct name for the medium-sized one; further analyses, including samples from other localities, are warranted to define the taxonomic status of the small species.
\end{abstract}

Keywords: Equus, Perissodactyla, Equidae, Pleistocene, El Cedral, México.

\section{RESUMEN}

En la literatura, ha habido mucha discusión sobre el número de especies de caballo, Equus (Perissodactyla, Equidae), que se pueden encontrar en una misma localidad del Pleistoceno de México, llegando a identificarse hasta 10 especies distintas. En este trabajo, se discute el número máximo de especies de Equus presentes en el Pleistoceno superior de la localidad arqueológica-paleontológica El Cedral, San Luis Potosí. Esta localidad está situada en una zona de matorral xerófilo de la llanura mexicana y es un depósito formado dentro de antiguos manantiales, que en ciertas épocas del año debieron dar lugar a lagunas. Fue excavado durante las décadas de los 70 y 80 y se observó una clara actividad de presencia humana alrededor de hace 30.000 años AP. Se obtuvo una fauna muy rica en mamíferos, más de 5.000 huesos fósiles, que incluye especies de ratones habitantes de bosques templados y un tapir de bosque tropical que no se conoce hasta la actualidad en ninguna localidad cercana. Hemos dispuesto de una muestra importante de restos de todas las partes del esqueleto, incluidos dientes y metápodos, que nos han permitido definir los rasgos más importantes de las formas de caballo de esta localidad. Se han realizado análisis estadísticos uni- y multivariantes cuyos resultados son discutidos en relación con la situación taxonómica de los distintos grupos obtenidos. La presencia de una forma de tamaño grande y otra pequeña es clara y quedan algunos restos entre ellas que no parecen corresponder a ninguna de las dos. Consideramos el nombre Equus mexicanus la asignación más apropiada para los caballos de tamaño grande, mientras que Equus conversidens parece ser el nombre correcto para los restos de talla intermedia. La determinación taxonómica de la especie de tamaño pequeño requiere de nuevos análisis, incluyendo restos de otras localidades, que se realizarán en un futuro trabajo.

Palabras clave: Equus, Perissodactyla, Equidae, Pleistoceno, El Cedral, México. 


\section{INTRODUCCIÓN}

En el pasado, ha habido mucha discusión acerca del número de especies de caballo del género Equus (Perissodactyla, Equidae) que existieron durante el Pleistoceno en México. En algunas localidades, se han identificado más de 10 especies (ver Mooser, 1958; Mooser y Dalquest, 1975). El objetivo principal de este trabajo es describir y determinar provisionalmente, en la medida de lo posible, los numerosos restos óseos de caballo procedentes del sitio arqueológico-paleontológico de El Cedral, San Luis Potosí, México. Para ello se estudian los caracteres morfométricos de los molares y elementos postcraneales $\mathrm{y}$, basándonos en el control estratigráfico disponible, establecemos cuantas especies pudieron coexistir temporalmente. Asimismo, se esbozan comparaciones con las formas conocidas de América del Norte y se infiere si hay algún tipo de relación con las que aparecen en América del Sur.

En México, los restos de caballo se han documentado desde mediados del siglo XIX. Owen (1869) describió la especie Equus conversidens a partir de fotografías de materiales procedentes de la Cuenca de México. Desde ese momento, diversos estudios se han abocado a reportar restos de caballos pleistocenos procedentes de diversas localidades distribuidas en la mayor parte de la República Mexicana (p. e., Álvarez, 1965; Dalquest, 1978, 1979; Gidley, 1901).

Álvarez y Ocaña (1987) sintetizaron el conocimiento nomenclatural referente al género Equus del Pleistoceno de México. Dichos autores indican que la enorme variación en la figura oclusal de los molares, que ha sido utilizada como base para las revisiones taxonómicas del género, ha propiciado la proliferación de especies y, por ende, de nombres disponibles. Para México, se han citado 26 especies, 12 de las cuales tienen su localidad típica en el país.

Recientemente, Reynoso-Rosales y Montellano-Ballesteros (1994) analizaron de manera cuantitativa el material fósil de caballos proveniente de la localidad Arroyo Cedazo, Aguascalientes, donde se han documentado hasta 11 especies. Al encontrar una mayor frecuencia de un tamaño y un patrón dental concluyeron que los équidos de dicha localidad pertenecen a una sola población, cuya variabilidad habría sido supraestimada, y a la vez proponen con ello intervalos de variación muy amplios, asignando todo el material a una sola especie, Equus conversidens.

Basada en un estudio multivariante de caracteres cuantitativos del cráneo, la mandíbula y los metápodos, Winans (1989) propuso que las especies del género Equus de América del Norte se pueden dividir en cinco grandes grupos: (1) E. simplicidens (Blanquense temprano); (2) $E$. scotti (Blanquense tardío a Rancholabreanense); (3) E. laurentius (Rancholabreanense); (4) E. francisci (Irvingtonianense a Rancholabreanense); y (5) E. alaskae
(Irvingtonianense a Rancholabreanense); sin embargo, la autora no decide si dichos grupos tendrían nivel específico o superior dentro de la jerarquía taxonómica y así, los define como grupo-especie.

\section{SITIO DE ESTUDIO}

El Rancho La Amapola se localiza a $1,7 \mathrm{~km}$ al sureste de El Cedral, San Luis Potosí (Fig. 1). Puntualmente queda a $23^{\circ} 49^{\prime} \mathrm{N}, 100^{\circ} 43^{\prime} \mathrm{O}$ y a una altitud de $1.700 \mathrm{~m}$. El área en general presenta varios yacimientos fosilíferos pleistocenos resultado de la presencia de manantiales, ahora secos, que en ciertas épocas del año formaban lagunas (Lorenzo y Mirambell, 1986, 1999). Hay que notar que la vegetación actual del área, matorral xerófilo, es muy distinta a la que existió en épocas pasadas, ya que por los fósiles existentes, hubo condiciones de mayor humedad, lo que propició las condiciones para la existencia de un pastizal (v.g., el ratón de campo o metorito Microtus pennsylvanicus (Ord, 1815) - Alvarez y Polaco, 1982; el mamut Mammuthus columbi (Farlconer, 1857) - ArroyoCabrales et al., 2003; y los caracoles terrestres Pupilla syngenes (Pilsbry, 1890), Grastrocopta pellucida (Pfeiffer, 1841) у Helicodiscus singleyanus (Pilsbry, 1890) - M. T. Olivera, comunicación personal, 2003) o incluso un bosque tropical caducifolio (Tapirus haysii (Leidy, 1860) Arroyo-Cabrales et al., 1996).

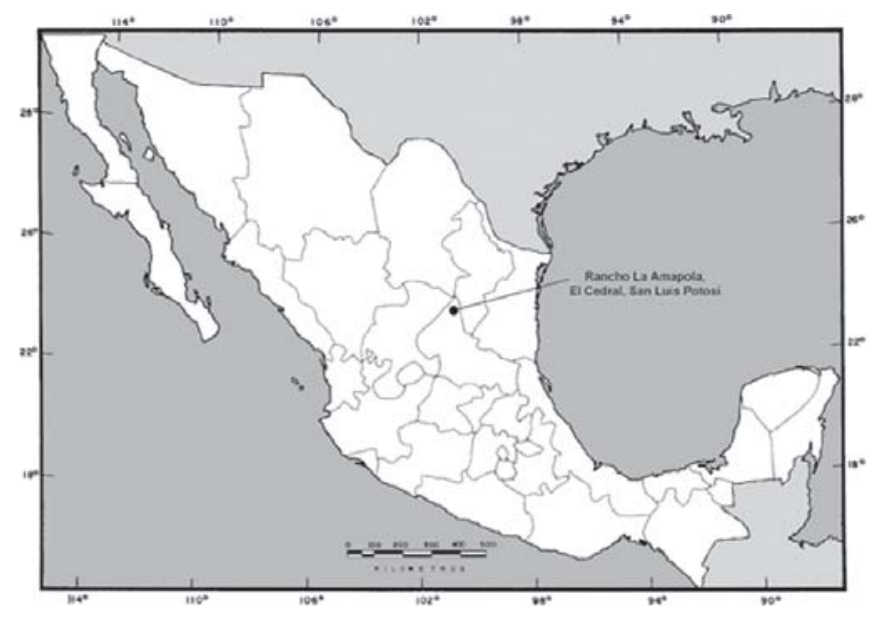

Figura 1. Situación geográfica de la localidad de Rancho La Amapola, a 1,7 km al sureste de El Cedral, San Luis Potosí, México.

Geographical location of the Rancho La Amapola site, at $1.7 \mathrm{~km}$ SE of El Cedral, San Luis Potosí, México.

De 1977 a 1984 y como parte del Proyecto "Cuencas Endorreicas" realizado por el personal del entonces Departamento de Prehistoria (hoy Subdirección de Laboratorios y Apoyo Académico) del Instituto Nacional de Antropología e Historia (INAH), se efectuaron investiga- 
ciones interdisciplinarias en el Rancho La Amapola. Los objetivos de dicho proyecto eran el mejor conocimiento de los asentamientos humanos del Pleistoceno tardío y Holoceno temprano desde una perspectiva ambiental.

En el sitio se localizaron varios hogares, comprendiendo un intervalo de entre 15 y 16 mil años; el más antiguo se halló en la capa XII, fechado en más de 35.000 años AP (37.694 \pm 1.963 años AP, Lorenzo y Mirambell, 1999), el más reciente fue fechado en $21.468 \pm 458$ años AP (capa X). Por otro lado, se hallaron diferentes horizontes faunísticos, es decir, ciertas capas con abundantes restos óseos generalmente muy

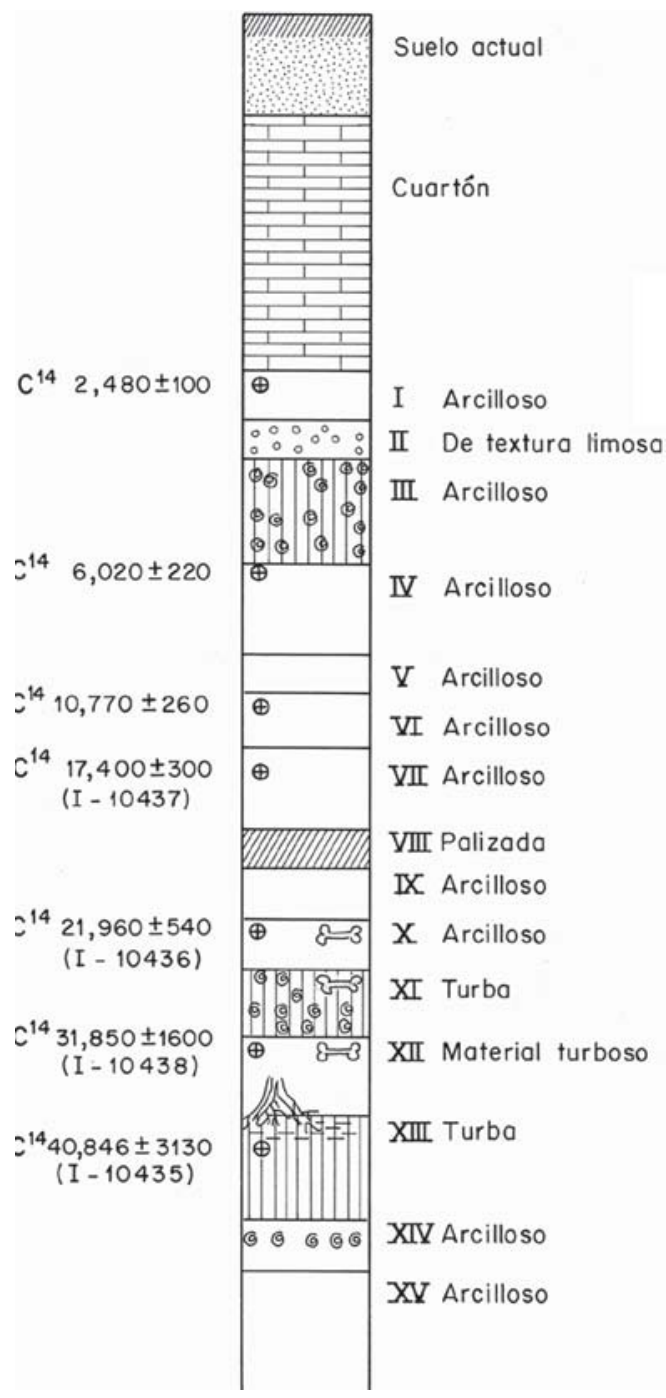

Figura 2. Esquema estratigráfico idealizado del sitio de El Cedral $(7,8 \mathrm{~m})$, con tres horizontes faunísticos de vertebrados. $\& 3$ : niveles con restos de Equus. Cuartón $=$ Duripan; Palizada representa restos de ramas y raíces de árboles.

Idealized stratigraphic section of the El Cedral site $(7,8 \mathrm{~m})$, with three vertebrate faunal levels. $\&_{-3}^{-}$: levels with Equus remains. Cuartón $=$ Dunipan $=$ Calcic duripan with calcic carbonate and gypsum. Palizada corresponds to brachs and roots of tree. fracturados, en pocos casos en relación anatómica y con una gran dispersión. Sin embargo, es notable la concentración de huesos de caballo entre las capas X y XII (Fig. 2).

Parte de la fauna ya ha sido documentada anteriormente, ya sea a manera de listado (Álvarez y Polaco,1981) o en estudios taxonómicos particulares (Álvarez y Polaco, 1982; Arroyo-Cabrales et al., 1996).

\section{MATERIAL Y MÉTODOS}

Los restos óseos recuperados durante ocho temporadas de excavación están depositados en la Colección Paleontológica del Laboratorio de Arqueozoología "M. en C. Ticul Álvarez Solórzano", Subdirección de Laboratorios y Apoyo Académico del Instituto Nacional de Antropología e Historia, México, D. F., y consisten en: 17 series yugales superiores, 11 series yugales inferiores, 137 dientes superiores aislados, 141 dientes inferiores aislados, siete fragmentos de escápula, 16 húmeros (uno entero), 26 radios (cuatro enteros), 25 metacarpianos terceros (MCIII), ocho metacarpianos segundos, 10 metacarpianos cuartos, 43 escafoides o naviculares del carpo, 33 magnos, 24 lunatum o semilunares, 18 piramidales o triquetum, 12 pisiformes, cuatro unciformes o $4^{\circ}$ carpal, cuatro $2^{\circ}$ carpal o trapezoides, seis fragmentos de pelvis, 11 fémures (uno entero), 23 tibias (tres enteras), una rótula, 64 astrágalos (AST), 28 calcáneos (CAL), 28 metatarsianos terceros (MTIII), cinco metatarsianos segundos, 15 metatarsianos cuartos, 32 naviculares del tarso, siete ectocuneiformes, 22 cuboides, $941^{\text {a }}$ falanges del dedo central (1FIII), $1022^{\text {a }}$ falanges del dedo central (2FIII), $343^{\text {a }}$ falanges del dedo central y 35 sesamoideos proximales. Además hay abundantes restos de vértebras, incisivos, caninos y fragmentos de distintos huesos que no hemos estudiado dado lo numeroso de la muestra.

Desde el punto de vista metodológico, las variables cuantificadas son las propuestas en la "Hipparion Conference" para el estudio de los équidos (Eisenmann et al., 1988). Todas las dimensiones van expresadas en milímetros.

En un primer estudio de los caballos realizado por Álvarez et al. (manuscrito en revisión), se sugirió la presencia en El Cedral de hasta cinco grupos (G1 a G5), tal vez representantes de distintas especies, por lo que hemos realizado un análisis de frecuencias que nos muestre la curva de distribución de las distintas dimensiones de cada uno de los huesos estudiados. A fin de comparar estos materiales con los procedentes de otras localidades americanas, hemos comparado nuestros datos con los suministrados por Winans $(1985,1989)$ sobre los caballos fósiles de América del Norte; asimismo, se ha realizado un análisis incluyendo los datos dentales y del esqueleto apendicular de las distintas especies de Equus (Amerhippus) de América del Sur (Prado y Alberdi, 1994; Alberdi y Frassinetti, 2000; Alberdi et al., 2003).

Se ha realizado un análisis de componentes principales (ACP) con el fin de explorar los posibles grupos (Fig. 3). Concretamente, los análisis los hemos llevado a cabo sobre una muestra de 94 1FIII, 102 2FIII, 64 AST, 28 CAL, 25 MCIII y 28 MTIII, así como en las muestras de huesos largos, 26 radios y 23 tibias, y en los dientes yugales sueltos: un total de 141 dientes inferiores y 137 dientes superiores. No fue necesaria una 
estandarización de los datos ya que sólo se consideran los caracteres continuos, es decir medidas expresadas en la misma escala. Este método, reduce sin gran pérdida de información, el número de dimensiones y de esa manera facilita la visualización de los huesos en función de los caracteres empleados. Estos componentes no están relacionados entre sí y por lo tanto se interpretan independientemente uno del otro. Cada componente contiene una parte de la variabilidad total de los caracteres. El primer componente es el que contiene mayor información y a menudo éste concentra la información sobre la talla de los caballos. Los resultados de esta técnica se graficaron en ejes ortogonales que representan los componentes principales. Otro método de ordenación que se utilizó fue el análisis discriminante (AD). En este análisis se utilizaron las distancias de Mahalanobis para la obtención de las matrices de similitud. En este caso se indican previamente los agrupamientos que se van a estudiar, el método consiste en maximizar las diferencias. A partir de los datos del ACP, se realizó el análisis discriminante con el fin de establecer el grado de significación de las diferencias entre los grupos (Fig. 4). Los detalles del método se pueden encontrar en Marcus (1990) y Reyment (1991). Se ha utilizado el paquete estadístico de SPSS 10.0.

\section{RESULTADOS}

El análisis de frecuencias, llevado a cabo con todas las dimensiones de las piezas más significativas del esqueleto apendicular y de los restos dentarios, ha permitido establecer la presencia de dos grupos claros, uno de gran tamaño (TG) y otro pequeño (TP), así como la presencia de algunos restos de talla intermedia (TM) entre los anteriores. La comparación con las formas de Equus (Amerhippus) Hoffstetter, 1950 de América del Sur no aportó resultados positivos.

En el caso de las series dentarias inferiores, observamos que tanto los premolares como los molares de talla grande están dentro de la variabilidad de lo que Winans (1989) llama grupo Equus laurentius. Sólo una serie premolar es algo más pequeña que las de este grupo, pero más grande que las dimensiones para el grupo E. alaskae de Winans. En el caso de las series superiores, nos encontramos que algunas dimensiones de las series premolar y molar son muy pequeñas como para corresponder a los datos del grupo E. alaskae, mientras que otras encajan con los datos de Winans (1989) para el grupo E. laurentius. De hecho, sólo una serie premolar de El Cedral, de talla pequeña, podría corresponder al grupo E. alaskae.

El análisis de componentes principales realizado indica que las variables que más contribuyen al componente1 son prácticamente todas, mientras que al componente2, sólo algunas longitudes contribuyen en el caso de los metápodos (Tabla 1). Esto ha permitido detectar igualmente la presencia de dos grupos claros de restos, aquellos que corresponden a un animal de tamaño grande y aquellos que corresponden a uno de tamaño pequeño. En el caso de los

\begin{tabular}{cccc}
\hline tipo hueso & componente & \% de la varianza & \%acumulado \\
\hline \multirow{2}{*}{ MCIII } & 1 & 86,945 & 86,945 \\
& 2 & 9,332 & 96,278 \\
\multirow{2}{*}{ MTIII } & 1 & 92,24 & 92,24 \\
& 2 & 5,087 & 97,327 \\
AST & 1 & 97,57 & 97,57 \\
& 2 & 0,864 & 98,433 \\
1FIII & 1 & 92,075 & 92,075 \\
& 2 & 4,277 & 96,351 \\
\multirow{2}{*}{ 2FIII } & 1 & 97,147 & 97,147 \\
& 2 & 1,602 & 98,749 \\
\hline
\end{tabular}

Tabla 1. Resultados de porcentaje de la varianza y porcentaje acumulado de los dos primeros componentes del análisis de componentes principales (ACP) de los restos fósiles de Equus de la localidad El Cedral: metacarpianos (MCIII), metatarsianos (MTIII), astrágalos (AST), primera falange del tercer dedo (1FIII) y segunda falange del tercer dedo (2FIII). Results of the Principal component analysis (PCA) of Equus fossil remains from $\mathrm{El} \mathrm{Cedral} \mathrm{site.} \mathrm{The} \mathrm{first}$ two principal components jointly contain per cent of the information accumulated and the variances: third metacarpal (MCIII), third metatarsal (MTIII), astragalus (AST), first phalanx of third digit (1PHIII), and second phalanx of third digit (2PHIII).

radios, hay un resto (DP-2975) que podría corresponder a un animal de tamaño pequeño, si bien corresponde a un animal joven, por lo que no lo hemos considerado. Es curioso observar en huesos tan numerosos, como los astrágalos, que los grupos previos, G5-G4 y G3, quedan claramente agrupados como animales de talla pequeña, mientras que, G1 y parte de G2 también quedan claramente agrupados como correspondientes a animales de talla grande. El resto de G2 corresponde a una talla intermedia. En el caso del MTIII, todos los restos quedan agrupados en dos grandes grupos correspondientes a animales de talla grande y talla pequeña, si bien entre los restos de talla pequeña se detecta la posibilidad de que a su vez hubiera dos grupos, uno grácil y otro robusto, lo que pudiera corresponder a diferentes taxa o bien a dimorfismo sexual (Fig. 3B). Las 1FIII, incluso diferenciando las anteriores de las posteriores, quedan claramente formando dos grandes grupos correspondientes a animales grandes y pequeños (Fig. 3D). En el caso de la 2FIII, la situación es similar, quedando dos grupos bien separados: G5-G4 y G3 de talla pequeña y G2 y G1 de talla grande, no quedando ningún resto de talla media (Fig. 3E). Entre los MCIII, sólo los ejemplares DP-4025 y DP-4681 corresponden a animales de tamaño medio; igualmente el cal- 

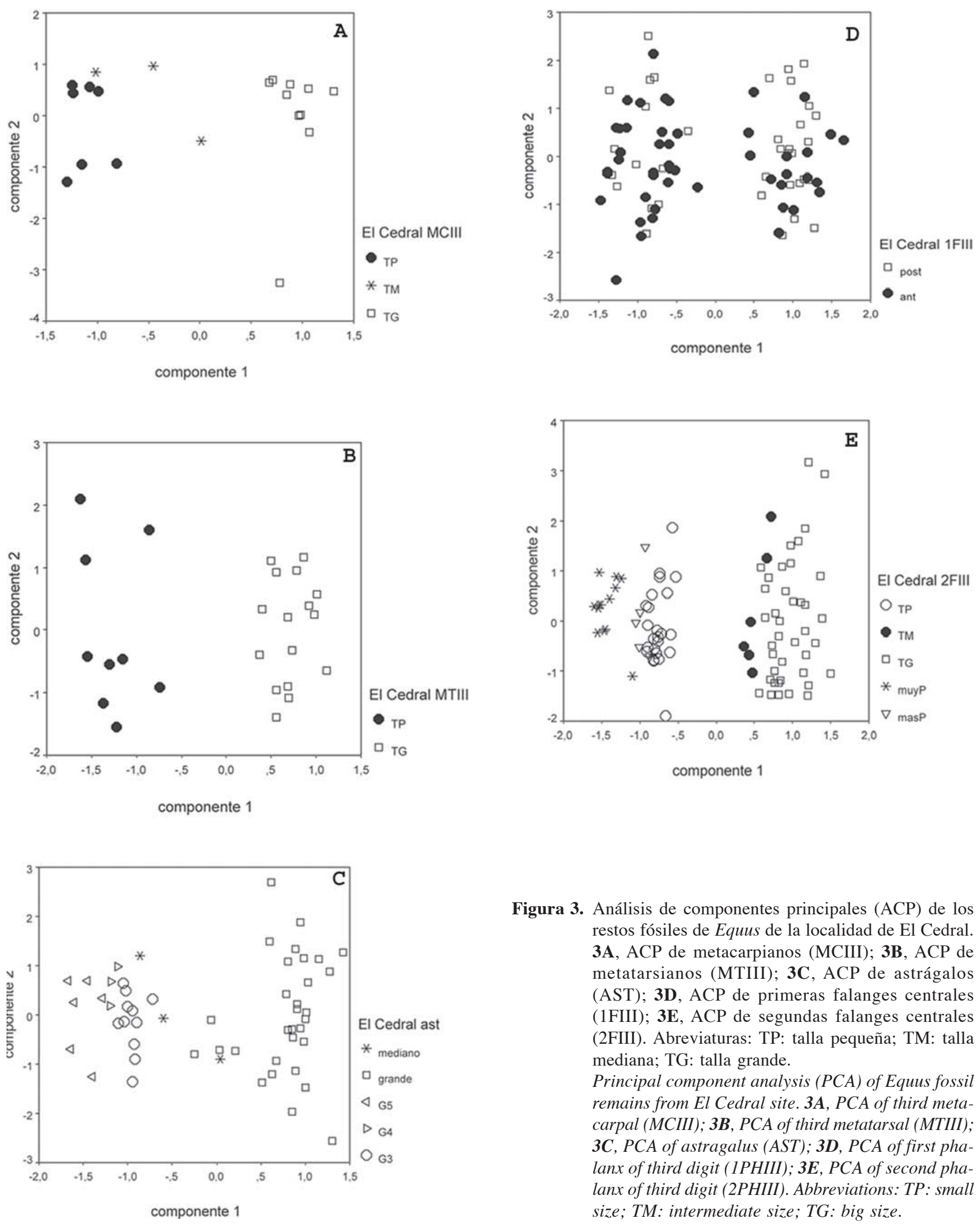

cáneo, DP-2823 y los astrágalos DP-2999, DP-4056, DP4064, DP-4684 y DP-4687 se podrían considerar como de este grupo intermedio.

Figura 3. Análisis de componentes principales (ACP) de los restos fósiles de Equus de la localidad de El Cedral. 3A, ACP de metacarpianos (MCIII); 3B, ACP de metatarsianos (MTIII); 3C, ACP de astrágalos (AST); 3D, ACP de primeras falanges centrales (1FIII); 3E, ACP de segundas falanges centrales (2FIII). Abreviaturas: TP: talla pequeña; TM: talla mediana; TG: talla grande.

Principal component analysis (PCA) of Equus fossil remains from $\mathrm{El}$ Cedral site. $3 \mathrm{~A}, \mathrm{PCA}$ of third metacarpal (MCIII); 3B, PCA of third metatarsal (MTIII); 3C, PCA of astragalus (AST); 3D, PCA of first phalanx of third digit (1PHIII); $3 E, P C A$ of second phalanx of third digit (2PHIII). Abbreviations: TP: small size; TM: intermediate size; TG: big size.

En el caso de la dentición, la situación es semejante y solamente algunos dientes aislados se ubican en una posición intermedia. 

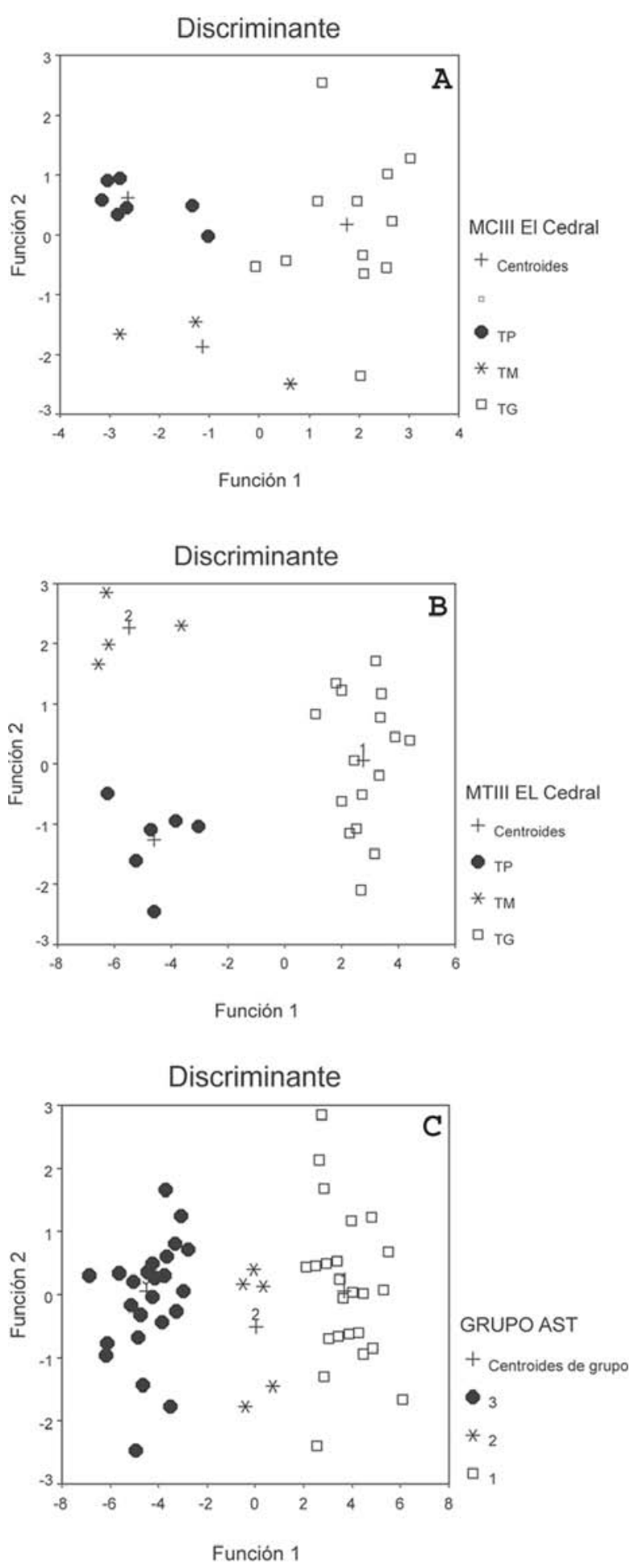

Los análisis discriminantes realizados confirman la mayor parte de los grupos detectados a partir de los análisis de componentes principales (Figs. 4A, 4B, 4C, 4D, 4E). El porcentaje de los casos originales correctamente clasificados es de: $95,5 \%$ en MCIII, $100 \%$ en MTIII y en AST, $98,9 \%$ en 1 FIII y $97,9 \%$ en 2 FIII.

Al comparar nuestros datos con los de Winans (1989), hemos detectado la posibilidad de establecer una cierta aproximación entre los équidos de El Cedral y algunas
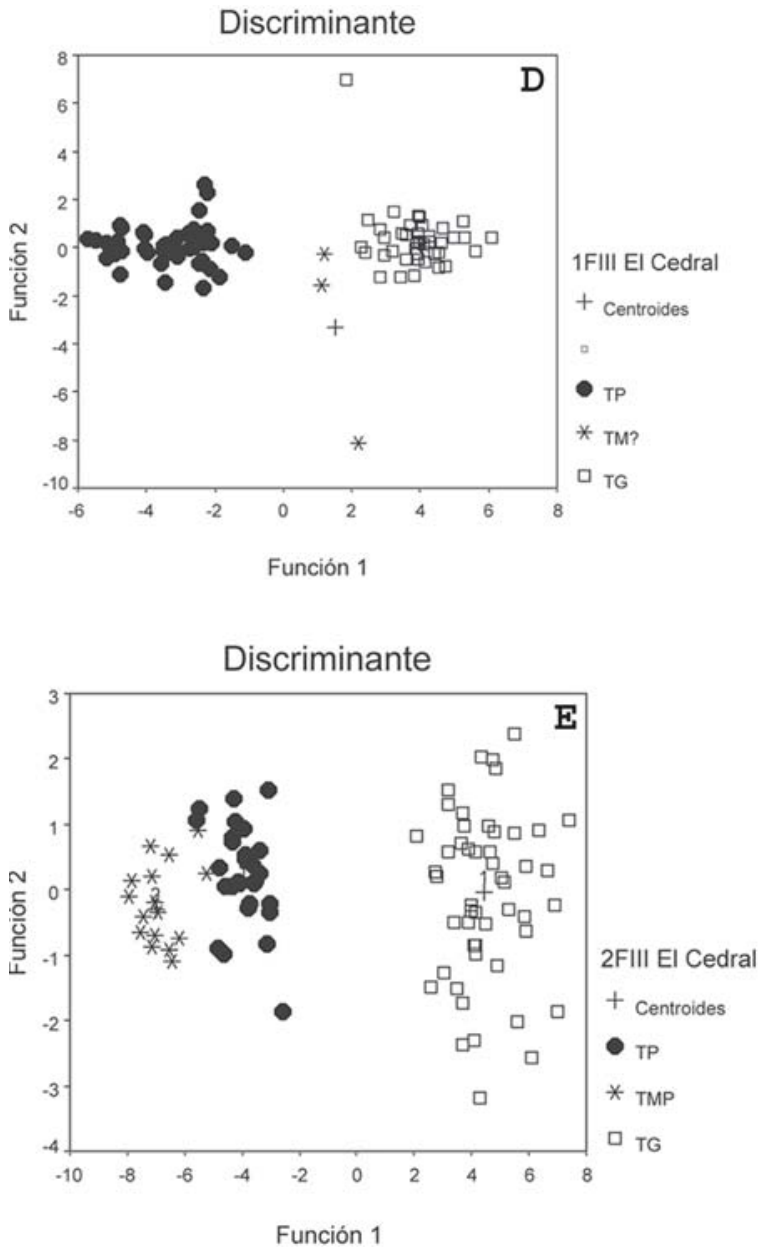

Figura 4. Análisis discriminante $(\mathrm{AD})$ de los distintos grupos que se forman en el ACP con respecto a los restos fósiles de Equus de la localidad de El Cedral. 4A, AD de metacarpianos (MCIII); 4B, AD de metatarsianos (MTIII); 4C, AD de astrágalos (AST); 4D, AD de primeras falanges centrales (1FIII); 4E, AD de segundas falanges centrales (2FIII). Abreviaturas como en fig. 3 y 1-2-3 representan los centroides. Discriminant analysis (DA) of the Equus remain groups recognized by $P C A$ from the El Cedral site. 4A, DA of third metacarpal (MCIII); $\mathbf{B B}, D A$ of third metatarsal (MTIII); 4C, DA of astragalus (AST); 4D, DA of first phalanx of third digit (1PHIII); $4 \boldsymbol{E}, D A$ of second phalanx of third digit (2PHIII). Abbreviations as in fig. 3, and 1-2-3 represent of centroids.

formas de América del Norte. Así, los metápodos de talla grande entran de lleno en la variación de los metápodos del grupo E. laurentius; incluso el índice entre la longitud y la anchura transversal máxima de los metacarpianos y los metatarsianos (menor de 5 y 6 , respectivamente) coincide con los restos procedentes de El Cedral. En el caso de las series dentarias de talla grande, también éstas entran en la distribución de las descritas por Winans para este grupo, si bien ella sólo detalla las series premolar y 
molar, pero no la longitud total de la serie. Esta autora incluye en el grupo de E. laurentius a E. mexicanus Hibbard, 1955, por ello pensamos que de manera provisional deberíamos asignar los restos de caballo de talla grande a E. mexicanus y como Winans no define taxonómicamente el grupo E. laurentius, sino que agrupa en él una serie de especies de Equus de América del Norte y los restos del esqueleto apendicular de El Cedral no se ajustan todos a la distribución del grupo E. laurentius como los restos de la dentición, queda pendiente un estudio más amplio y exhaustivo que nos permita definir exactamente las relaciones entre estos restos y el grupo $E$. laurentius de Winans (1989).

La comparación de los restos de talla pequeña es algo más complicada. Winans (1989) agrupa los caballos de talla pequeña con metápodos robustos en el grupo $E$. alaskae y a los caballos con metápodos gráciles en el grupo E. francisci. Al comparar nuestros datos, observamos que los restos de El Cedral son, en general, menores que los datos de Winans, aunque hay un solapamiento con los valores más pequeños tanto del grupo E. alaskae como del grupo E. francisci. De este análisis, nosotros deducimos que los grupos de Winans (1989) resultan un tanto heterogéneos, sobre todo en el caso del grupo E. francisci. Esto nos inclina a pensar que el caballo pequeño de El Cedral podría corresponder a una especie distinta de las estudiadas por Winans (1989) para Estados Unidos y hasta su estudio detallado la denominaremos como Equus sp. A.

Por último, los escasos restos que hemos señalado como de talla mediana, que en la colección están asignados a $E$. conversidens Owen, 1869, se sitúan dentro de la variabilidad del grupo E. alaskae, grupo en el que esta autora incluye a E. conversidens. Los dos restos de MCIII asignados a E. conversidens estarían próximos a los dados por Winans para el grupo E. alaskae pero son un poco más pequeños y en el caso de las series dentarias hay una serie premolar superior (DP-2679-2678-2677 d) y otra inferior (DP-2633 d) que se sitúan en el intervalo del grupo E. alaskae. Sin embargo, como hemos apuntado más arriba, la taxonomía que aporta Winans (1989) es aproximativa y el estudio definitivo de las especies representadas en la localidad de El Cedral, precisa de una revisión de las formas norteamericanas, lo que excede los límites de este trabajo.

Nuestros datos podrían confirmar la presencia de un máximo de tres especies de caballo en la localidad de El Cedral, una que podría relacionarse con el grupo Equus laurentius y que denominamos provisionalmente como Equus mexicanus. Esta especie está bien representada en el yacimiento de El Cedral tanto por los restos del esqueleto apendicular como por los de la dentición.

Con dudas podríamos asignar al grupo E. alaskae algunos escasos restos del esqueleto apendicular y de la dentición, que se sitúan dentro de su variabilidad y que en la colección están asignados a E. conversidens. Estos restos aunque escasos parecen ser un poco más grandes y más robustos que los restos que se han agrupado como de talla pequeña.

Los restos del esqueleto y la dentición de talla pequeña de El Cedral son bastante más pequeños y con metápodos más gráciles que los del grupo $E$. alaskae y/o $E$. francisci. Para la determinación taxonómica detallada y definitiva de estos restos, es necesaria una comparación exhaustiva con todos los restos de caballo de talla pequeña que se hayan encontrado en América del Norte, de manera general y, de manera particular, en América Central. Asimismo, la diferencia de gracilidad entre los MTIII pequeños de El Cedral conduce a la hipótesis de la existencia de un cierto dimorfismo sexual en la forma pequeña.

\section{CONCLUSIONES}

Los estudios llevados a cabo con los caballos fósiles encontrados en la localidad de El Cedral, así como los análisis de ${ }^{14} \mathrm{C}$ realizados (ver Lorenzo y Mirambell, 1986), nos permiten emplazar esta localidad en el Pleistoceno superior. Por otra parte, el estudio morfológico indica que sólo pueden detectarse diferencias de talla, ya que la morfología tanto dentaria como del esqueleto postcraneal es similar en todos los restos examinados. Los análisis de componentes principales y los discriminantes permiten asegurar la presencia de al menos dos especies distintas de Equus. Una incluye todos los restos que hemos denominado como de talla grande (TG) y que asignamos provisionalmente a E. mexicanus. La segunda, donde incluimos todos los restos que hemos denominado como de talla pequeña (TP) queda identificada preliminarmente como Equus sp. A. Hay una serie de restos, muy escasos, de un caballo de talla intermedia (TM) en relación a los otros dos, que asignamos provisionalmente a E. conversidens.

\section{AGRADECIMIENTOS}

Agradecemos a la Profa. Lorena Mirambell, encargada de las investigaciones arqueológicas en el sitio de El Cedral, San Luis Potosí, la autorización para utilizar en la publicación los resultados del estudio de materiales de dicha localidad aún sin documentar; asimismo, agradecemos a las autoridades del Museo Nacional de Ciencias Naturales, CSIC, Madrid, España, y a la Subdirección de Laboratorios y Apoyo Académico del Instituto Nacional de Antropología e Historia, México, su continuo apoyo para la realización de esta investigación. La Biól. Felisa J. Aguilar amablemente preparó el mapa de la figura 1 y el Sr. Guillermo Herrera dibujo el corte estratigráfico de la figura 2. Asimismo, agradecemos a los Drs. Esperanza Cerdeño y Paul Palmqvist la revisión del manuscrito. Este trabajo ha sido posible gracias al Convenio Bilateral CSIC-CONACYT (Proyecto 2001MX0010) durante los años 2001 y 2002; así como a los proyectos PB97-1250 y BTE2001-1864 de la DGICYT de España. 


\section{BIBLIOGRAFÍA}

Alberdi, M.T., Cartelle, C. y Prado, J.L. 2003. El Registro Pleistoceno de Equus (Amerhippus) e Hippidion (Mammalia, Perissodactyla) de Brasil. Consideraciones Paleoecológicas y Biogeográficas. Ameghiniana, 40, 173-196.

Alberdi, M.T. y Frassinetti, D. 2000. Presencia de Hippidion y Equus (Amerhippus) (Mammalia, Perissodactyla) y su distribución en el Pleistoceno superior de Chile. Estudios geológicos, 56, 279-290.

Álvarez, T. 1965. Catálogo paleomastozoológico mexicano. Instituto Nacional de Antropología e Historia, México, Departamento de Prehistoria, Publicaciones, 17, 1-70.

Álvarez, T. y Ocaña, A. 1987. Nomenclatura específica de los Equidae del Pleistoceno de México. Anales de la Escuela Nacional de Ciencias Biológicas, México, 31, 125-132.

Álvarez, T. y Polaco, O.J. 1981. Anexo 1. Fauna obtenida de las excavaciones realizadas en el sitio Rancho La Amapola - El Cedral, S.L.P. In: El Cedral, S.L.P., México: Un sitio con presencia humana de más de 30,000 AP. (Eds. J.L. Lorenzo y L. Mirambell). X Congreso de la Unión Internacional de Ciencias Prehistóricas y Protohistóricas, México, 1981, 123-124.

Álvarez, T. y Polaco, O.J. 1982. Restos pleistocénicos de dos especies de Microtus (Rodentia: Muridae), del norte de San Luis Potosí, México. Anales de la Escuela Nacional de Ciencias Biológicas, México, 26, 47-53.

Arroyo Cabrales, J., Polaco, O.J., Álvarez ,T. and Jonson, E. 1996. New records of fossil tapir from northeastern México. Current Research in the Pleistocene, 13, 93-95.

Arroyo-Cabrales, J., Polaco, O.J., Johnson, E and Guzmán, A.F. 2003. The distribution of the genus Mammuthus in Mexico. In: Advances in mammoth research (Proceedings of the Second International Mammoth Conference, Rotterdam, May 16-20 1999) (Eds. J. W. F. Reumer, J. De Vos and D. Mol). Deinsea, 9, 27-39

Dalquest, W.W. 1978. Phylogeny of American horses of Blancan and Pleistocene age. Annals of Zoologia Fennica, 15, 191-199.

Dalquest, W.W. 1979. The little horses (genus Equus) of the Pleistocene of North America. American Midland Naturalist, 101, 241-244.

Eisenmann, V., Alberdi, M.T., De Giuli, C. and Staesche, U. 1988. Collected papers after the "New York International Hipparion Conference, 1981". In: Studying fossil horses, 1, Methodology (Eds. M. Woodburne and P. Sondaar). E.J. Brill, Leiden, 1-72.
Gidley, J.W. 1901. Tooth characters and revision of the North American species of the genus Equus. Bulletin of the American Museum of Natural History, 14, 91-141.

Lorenzo, J.L. and Mirambell, L. 1986. Preliminary report on archaeological and paleoenvironmental studies in the area of El Cedral, San Luis Potosí, México, 1977-1980. In: New evidence for the Pleistocene peopling of the Americas (Ed. A.L. Bryan). Center for the Study of the Early Man, University of Maine, Orono, Peopling of the Americas series, 107-113.

Lorenzo, J.L. and Mirambell, L. 1999. The inhabitants of México during the Upper Pleistocene. In: Ice age peoples of North America. Environments, origins, and adaptations of the First Americans (Eds. R. Bonnichsen and K. L. Turnmire). Center for the Study of the First Americans, Oregon State University Press, Corvallis, 482-496.

Marcus, L.F. 1990. Traditional morphometrics. In: Proceedings of the Michigan Morphometrics Workshop (Eds. F.J. Rohlf and F.L. Bookstein). University of Michigan, Museum of Zoology, Special Publication 2, 78-122.

Mooser, O. 1958. La fauna "Cedazo" del Pleistoceno en Aguascalientes. Anales del Instituto de Biología Mexicano, 29, 409-452.

Mooser, O. and Dalquest, W.W. 1975. Pleistocene mammals from Aguascalientes, Central México. Journal of Mammalogy, 56, 781-820.

Owen, R. 1869. On fossil remains of Equines from Central and South America referable to Equus conversidens Ow. E. tau Ow., and E. arcidens Ow. Philosophical Transactions of the Royal Society of London, 159, 559-573.

Prado, J.L. and Alberdi, M.T. 1994 . A quantitative review of the horse Equus from South America. Palaeontology, 37, 459-481.

Reyment, R.A. 1991. Multidimensional paleobiology. Pergamon Press, 416 pp.

Reynoso-Rosales, V.H. y Montellano-Ballesteros, M. 1994. Revisión de los équidos de la Fauna Cedazo del Pleistoceno de Aguascalientes, México. Revista Mexicana de Ciencias Geológicas, 11, 87-105.

Winans, M.C. 1985. Revision of North American fossil species of the genus Equus (Mammalia: Perissodactyla: Equidae). Ph. D. Dissertation, University of Texas, Austin.

Winans, M.C. 1989. A quantitative study of North American fossil species of the genus Equus. In: The Evolution of Perissodactyls (Eds. D.R. Prothero and R.M. Schoch). Oxford University Press, 263-297.

Manuscrito recibido: 14 de abril, 2003 Manuscrito aceptado: 3 de septiembre, 2003 